\title{
SUPERIOR MESENTERIC ARTERY SYNDROME AS A RARE GASTROINTESTINAL MANIFESTATION OF SYSTEMIC LUPUS ERYTHEMATOSUS: CASE REPORT
}

Renata Borges de Lima ${ }^{1, \star}$, Gustavo Luiz Behrens Pinto ${ }^{1}$, Maria de Lourdes Castro de Oliveira Figueiroa ${ }^{1}$, Viviane Leal Novais ${ }^{1}$, Alisson Regis de Santana ${ }^{1}$, Mariana Oliveira Miranda ${ }^{1}$, Victor Pereira Mattos ${ }^{1}$, José Simões do Nascimento Neto ${ }^{1}$, Rebeca Souza Sessa Dantas ${ }^{1}$, Mittermayer Barreto Santiago ${ }^{1}$

1.Universidade Federal da Bahia, Salvador (BA), Brazil.

*Corresponding author: reblima1@gmail.com

\section{BACKGROUND}

Systemic lupus erythematosus (SLE) is a systemic autoimmune disease associated with gastrointestinal manifestations. Among them, lupus enteritis, pancreatitis, intestinal pseudo-obstruction and protein-losing enteropathy stand out. We present a case of a female SLE patient with intestinal obstruction due to superior mesenteric artery syndrome (SMAS).

\section{CASE REPORT}

A 20-year-old female SLE patient was admitted for investigation in April 2020 with vomiting, pain and abdominal distension for one week, evolving with complete intestinal obstruction. On physical examination, she was emaciated and dehydrated, with a distended abdomen and painful palpation. The rest of the physical examination was unremarkable. Laboratory tests were without significant changes. Computed tomography (CT) of the abdomen was performed and showed marked gastric distension (measuring $20.0 \times 8.0 \times 26.0 \mathrm{~cm}$, with an estimated volume of $2163 \mathrm{~mL}$ ) and part of the duodenum with an abrupt reduction in caliber in the third duodenal portion without mechanical obstructive factor identified by the method (Figures 1 and 2). It also showed a reduction in the aortomesenteric angle, coinciding with the site of luminal narrowing of the third portion of the duodenum in a previous examination, suggesting superior mesenteric artery syndrome (Figure 3). The general surgery team was activated, with a videolaparoscopic duodenojejunostomy performed uneventfully, with complete resolution of symptoms.

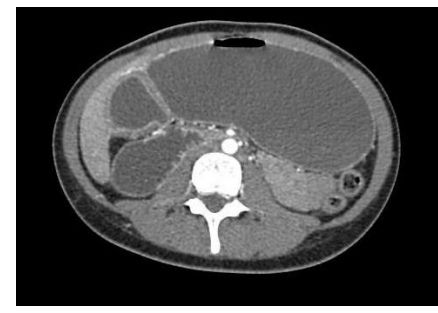

Figure 1.

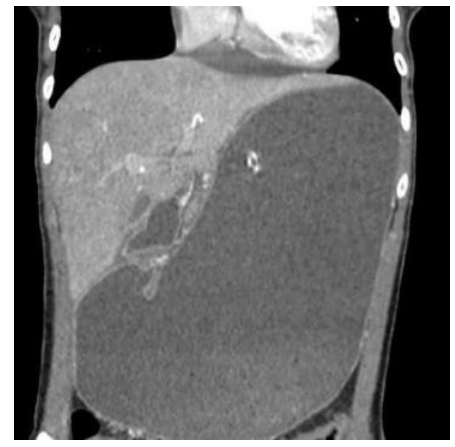

Figure 2.

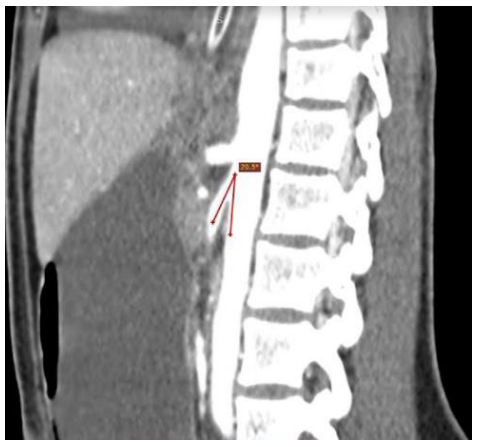

Figure 3.

\section{CONCLUSION}

Gastrointestinal symptoms are common in SLE patients and may be present in up to $50 \%$ of patients. Among them, abdominal pain, nausea, vomiting and diarrhea stand out, which may be associated with protein-losing enteropathy, intestinal pseudo-obstruction, liver involvement and pancreatitis. The SMAS is a potentially serious condition that manifests itself through symptoms of high intestinal obstruction and is characterized by a reduction in the aortomesenteric angle due to a reduction in the fat pad in this region. It is associated with debilitating conditions that lead to rapid and progressive weight loss, such as malignancies, malabsorptive syndromes, trauma and extensive burns. Treatment is based on nutritional support and, in refractory cases, surgical correction with duodenojejunostomy. There is only one previous report in the literature on the association of SLE and SMAS, which developed due to weight reduction in the initial phase of the underlying disease. The existence of a pathophysiological relationship between these conditions remains unclear. We should be aware of this complication in SLE patients who develop symptoms of gastrointestinal obstruction.

\section{KEYWORDS}

Systemic lupus erythematosus, Mesenteric artery, Superior, Gastrointestinal diseases. 